\#119 Resistência adesiva ao esmalte de materiais utilizados como plano de mordida em Ortodontia

Ricardo Sá Rodrigues*, Pedro Dias, Américo Ferraz, Jaime Portugal, Afonso Pinhão Ferreira, Maria João Ponces

Faculdade de Medicina Dentária da Universidade de Lisboa; Faculdade de Medicina Dentária da Universidade do Porto

Objetivos: Avaliar e comparar a resistência adesiva ao esmalte de diversos materiais utilizados para a confeção de planos de mordida em Ortodontia.

Materiais e métodos: A partir de 40 molares humanos, foram obtidos 80 discos com superfície em esmalte que foram distribuídos de forma aleatória por 4 grupos experimentais $(\mathrm{n}=20)$ criados de acordo com o material utilizado (Spectrum TPH3; Twinky Star; OptiBand Ultra; GC Fuji Ortho Band LC). Nos espécimes fabricados com Spectrum TPH3 e Twinky Star, antes da aplicação do material de plano de mordida, o esmalte foi condicionado com ácido fosfórico a 37\% durante 30 segundos, e após lavagem e secagem, foi aplicada uma camada fina de adesivo (Retensin Plus). O OptiBand Ultra e o GC Fuji Ortho Band LC foram aplicados diretamente sobre o esmalte, sem pré-condicionamento. A fotopolimerização do material de plano de mordida foi realizada com o aparelho LED, sendo que a cada $2 \mathrm{~mm}$ de material se fez uma polimerização de 20s (1200 $\mathrm{mW} / \mathrm{cm} 2$ ). Os espécimes foram então armazenados em saliva artificial e, de seguida, colocados em banho maria a aproximadamente $37 .{ }^{\circ} \mathrm{C}$ num período de $65 \mathrm{~h}$. Os testes de resistência adesiva foram realizados (Instron; $1 \mathrm{~mm} / \mathrm{min}$; $1 \mathrm{KN}$ ) submetendo os espécimes a tensões de corte até à fratura. Foram considerados os valores de 5,9 $\mathrm{MPa}$ e 7,8 $\mathrm{MPa}$ como valores mínimos que um plano de mordida deverá atingir para obter um desempenho clínico aceitável. Os resultados foram analisados estatisticamente com o ANOVA e testes post-hoc, segundo o método de Tukey (alfa=0,05).

Resultados: Os valores de resistência adesiva média obtidos foram de: 8,6 MPa para o OptiBand Ultra; 10,6 MPa para o GC Fuji Ortho Band LC; 14,8 MPa para o Spectrum TPH3; e, 17,1 MPa para o Twinky Star. Não se observaram diferenças estatisticamente significativas entre o OptiBand Ultra e o GC Fuji Ortho Band LC ( $p=0,805)$, entre o GC Fuji Ortho Band LC e o Spectrum TPH3 $(p=0,271)$, nem entre o Spectrum TPH3 e o Twinky Star $(p=0,763)$.

Conclusões: Os quatros materiais atingiram, nas condições testadas, os valores de resistência adesiva ao esmalte de referência para um desempenho clínico aceitável como plano de mordida.

http://doi.org/10.24873/j.rpemd.2018.11.425

\#120 o cimento - influência no resultado final da reabilitação em Prótese Fixa

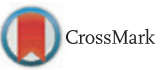

Joana Piloto*, Cláudia Volpato, Paulo Rocha Almeida, Pedro Mesquita, JC Sampaio Fernandes, Paula Vaz

UFSC, FMDUP

Objetivos: Pretendeu-se investigar a influência da cor do cimento de resina na cor final de uma restauração estética de prótese fixa. Considerou-se como hipótese nula: a cor do cimento de resina não influencia a cor final da reabilitação em zircónia, nas condições testadas e respetivos materiais utilizados neste estudo in vitro.

Materiais e métodos: No intuito de simular a superfície dentinária como base da restauração final, prepararam-se vinte amostras de resina composta $\left(\mathrm{Voco}^{\circledR}\right)$, em forma de disco, com 2,5 mm de espessura. Como material restaurador selecionou-se a zircónia (Zirlux ${ }^{\circledR}$ ), produzindo-se vinte e cinco discos com 1,0 mm de espessura, dos quais cinco representavam a cor preliminar no estudo (controlo). Para a cimentação adesiva de cada disco de resina composta com um de zircónia, utilizaram-se duas cores de cimento de resina $\left(\right.$ Bifix $^{\circledR} \mathrm{SE}$, cor universal e Bifix ${ }^{\circledR} \mathrm{SE}$, cor branco-opaco). Obtiveram-se dois grupos U e B $(n=10)$ cada um relativo a uma cor de cimento, que constituíram a amostra final deste estudo. Foi efetuada uma análise da cor da amostragem e de discos controlo, através do sistema CIE $\mathrm{L}^{*} \mathrm{a}^{*} \mathrm{~b}^{*}$, com espectrofotómetro (CM-3700d, Konica Minolta ${ }^{\circledR}$, Japão), calibrado e preparado. A variação da cor ( $\triangle \mathrm{E} 00)$ foi calculada. Efetuou-se uma análise estatística através do One-way ANOVA $(\mathrm{p}<0,001)$ e diversas comparações por análise de variância multifatorial.

Resultados: Não se obtiveram diferenças estatisticamente significativas (valor $\mathrm{p}>0,001$ ) para $\Delta \mathrm{E}, \Delta \mathrm{L}^{\prime}, \Delta \mathrm{C}^{\prime}$ e $\Delta \mathrm{H}^{\prime}$, entre a amostra e o grupo de controlo, aceitando-se a hipótese nula. Independentemente da cor docimento, a cor final da amostra, na reabilitação com zircónia, não variou significativamente da cor preliminar do grupo de controlo.

Conclusões: Dentro das limitações deste estudo, a cor da reabilitação em zircónia não parece ter sido influenciada pela cor do substrato. Torna-se emergente investir em novos estudos, que envolvam outras variáveis, ou por ventura a mesma, mas sujeita a materiais diferentes, ou com outra seleção de cores. http://doi.org/10.24873/j.rpemd.2018.11.421

\section{\#121 Veiculação De Clorexidina Em Resinas Acrílicas: Atividade Antibiofilme E Citotoxicidade}

Joana Vieira Costa*, Isabel Ribeiro, Lídia Gonçalves, Ana Bettencourt, Jaime Portugal, Cristina Bettencourt Neves

Faculdade de Farmácia da Universidade de Lisboa; Faculdade de Medicina Dentária da Universidade de Lisboa

Objetivos: Avaliar a atividade antibiofilme contra Candida albicans e o potencial citotóxico, utilizando culturas de fibroblastos, de três resinas acrílicas de rebasamento contendo clorexidina.

Materiais e métodos: Concentrações distintas de clorexidina foram selecionadas para inclusão na composição de três resinas acrílicas de rebasamento. Kooliner foi incorporado com 2,5\% (m/m) enquanto que Ufi Gel Hard e Probase Cold com 5\% $(\mathrm{m} / \mathrm{m})$. Todos os materiais também incluíram um grupo controlo ( $0 \%$ clorexidina). Para determinar a atividade antibiofilme, discos de resina foram colocados em meio inoculado com Candida albicans (ATCC 10231) e incubados durante 48 horas a $37^{\circ} \mathrm{C}$. Os espécimes com biofilme à superfície foram fixados com diferentes soluções de etanol, analisados e fotografados através de um microscópio eletrónico de varrimento (SEM). 
Para determinar o potencial citotóxico, foram obtidos extratos através da incubação dos espécimes $(n=2)$ em $1 \mathrm{~mL}$ de água destilada durante 24 horas a $37^{\circ} \mathrm{C}$. Posteriormente, as culturas de fibroblastos L929 (ATCC1 CCL-1TM) foram expostas aos extratos e a viabilidade celular foi determinada pelo ensaio espectrofotométrico de redução do brometo de tetrazólio (MTT). Foram utilizados grupos de controlo negativo e positivo ao ensaio. Como complemento, foi determinado o IC50 (concentração necessária para inibir $50 \%$ da viabilidade celular) da clorexidina. Os resultados foram analisados estatisticamente com testes paramétricos t-test (alfa $=0,05)$.

Resultados: A análise qualitativa das imagens obtidas por microscopia eletrónica de varrimento demonstrou a existência de uma camada de biofilme de Candida albicans em todos os grupos, excetuando-se Probase Cold com 5\% de clorexidina no qual não se observou qualquer microrganismo na superfície do material. A incorporação do fármaco diminuiu a viabilidade celular nas três resinas acrílicas em estudo $(p<0,001)$. Quando comparadas entre si, Probase Cold foi a resina menos citotóxica $(70,6 \pm 6,17 \%)$ e Ufi Gel Hard a mais citotóxica $(16,6 \pm 5,24$ $\%)$. O IC50 da clorexidina para a cultura celular utilizada foi igual a $8,0 \pm 1,10 \mu \mathrm{g} / \mathrm{mL}$.

Conclusões: O Probase Cold revelou ser a resina acrílica de rebasamento com maior atividade antibiofilme contra Candida albicans. Relativamente ao potencial citotóxico, as três resinas incorporadas com clorexidina diminuíram a viabilidade celular quando em contacto com a cultura de fibroblastos comparativamente aos grupos controlo, sendo Probase Cold a resina menos citotóxica.

http://doi.org/10.24873/j.rpemd.2018.11.424

\#122 Efeito da contaminação salivar e do método de descontaminação na adesão à dentina CrossMark

Joana Luís*, Margarida Venancio, Sara Gomes, Ana Filipa Chasqueira, Jaime Portugal

Faculdade de Medicina Dentária da Universidade de Lisboa

Objetivos: Avaliar a influência da contaminação salivar após polimerização de um adesivo universal e comparar a eficácia de dois diferentes métodos de descontaminação de saliva e da reaplicação do adesivo, na resistência adesiva à dentina, após 24 horas e 6 meses.

Materiais e métodos: A partir de 50 molares humanos íntegros foram obtidos 100 discos de dentina que foram distribuídos aleatoriamente por 10 grupos experimentais de acordo com as várias combinações possíveis entre protocolo adesivo [sem contaminação (SC); descontaminação com água (A); descontaminação com água seguida de reaplicação do adesivo (A Ad); descontaminação com etanol (E), descontaminação com etanol seguida de reaplicação do adesivo (E Ad)], e tempo de envelhecimento após adesão [24 horas $(24 \mathrm{H}) ; 6$ meses $(6 \mathrm{M})]$. Todos os espécimes foram restaurados com Scotchbond Universal Adhesive e Tetric EvoCeram. Após o respetivo período de envelhecimentos, os espécimes foram submetidos ao teste de resistência adesiva a tensões de corte $(1 \mathrm{~mm} / \mathrm{min}$; $1 \mathrm{KN})$ e a falha classificada em adesiva, coesiva ou mista. Os resultados de resistência adesiva obtidos foram sujeitos a testes estatísti- cos não-paramétricos de acordo com o método Kruskal-Wallis e Mann-Whitney (alfa=0,05). Foi utilizado o teste de Qui-quadrado para a análise estatística do tipo de falha (alfa=0,05).

Resultados: Os valores médios de resistência adesiva variaram entre os 38,5 MPa para o grupo SC-24H e os 3,1 MPa para os grupos A-6M e E-6M. Foi observada uma diminuição estatisticamente significativa $(\mathrm{p}<0,001)$ da resistência adesiva com o aumento do tempo de envelhecimento. Tanto para as $24 \mathrm{H}$ como para os $6 \mathrm{M}$, a contaminação com saliva resultou numa diminuição estatisticamente significativa $(\mathrm{p}<0,05)$ dos valores de resistência adesiva. Não se observaram diferenças significativas $(p>0,05)$ entre os dois métodos de descontaminação (água vs. etanol). Aos 6M, a reaplicação do adesivo permitiu obter valores de resistência adesiva mais elevados que sem reaplicação ( $p<0,001)$. O tipo de falha de união foi predominantemente do tipo adesivo e não se observaram diferenças $(p=0,667)$ entre os grupos experimentais.

Conclusões: A contaminação salivar após a polimerização do adesivo universal diminuiu os valores de resistência adesiva. A reaplicação do adesivo após descontaminação, apesar de não recuperar as forças de adesão, parece ser aconselhável. http://doi.org/10.24873/j.rpemd.2018.11.356

\#123 Efeito de tratamentos térmicos pós-polimerização na estabilidade cromática do bis-acrílico

Daniela Lourenço *, Maria Santos, Cláudia Semião, Maria Inês Pinto, Bruno Seabra, Jaime Portugal

Faculdade de Medicina Dentária da Universidade de Lisboa

Objetivos: Avaliar o efeito de vários tratamentos térmicos pós-polimerização na estabilidade cromática de duas resinas bis-acrílicas, quando imersas em café durante 24 horas e 7 dias.

Materiais e métodos: Foram fabricados 100 discos de resina bis-acrílica e distribuídos por 10 grupos experimentais $(n=10)$ de acordo com as combinações possíveis entre a resina bis-acrílica [Structur 3; Protemp 4] e o tratamento térmico pós-polimerização [sem tratamento (nt); microondas a $750 \mathrm{~W}$ durante 1 minuto (mw1); imersão em água a $60^{\circ} \mathrm{C}$ durante $1 \mathrm{mi}$ nuto (wb1); imersão em água a $60{ }^{\circ} \mathrm{C}$ durante 5 minutos (wb5); calor produzido por secador de cabelo convencional a $1800 \mathrm{~W}$ a $20 \mathrm{~cm}$ a $60^{\circ} \mathrm{C}$ (hd1)]. Trinta minutos após o início da manipulação das resinas, foi realizada a medição inicial da cor dos espécimes que em seguida foram imersos numa solução de café. As medições foram realizadas com um espectrofotómetro Easyshade de acordo com o sistema CIElab. Foram realizadas novas medições após 24 horas e 7 dias de armazenamento e o $\triangle \mathrm{E}$ foi calculado. Os dados de $\Delta \mathrm{E}$ foram analisados estatisticamente com ANOVA e testes post-hoc segundo Tukey (alfa=0,05).

Resultados: $O \triangle E$ variou entre 5,9 e 24,7. O Structur 3 apresentou um $\triangle E$ estatisticamente $(p<0,001)$ mais elevado que o Protemp 4, após os 2 períodos de envelhecimento. $\mathrm{O} \Delta \mathrm{E}$ foi influenciado estatisticamente $(p<0,001)$ pelo tratamento térmico pós-polimerização, tanto às 24 horas como aos 7 dias. Para o Structur 3, os grupos com tratamento térmico não apresentaram diferenças estatísticas $(p>0,05)$ entre si e obtiveram todos valores médios de $\Delta E$ estatisticamente $(p<0,05)$ superio- 